Volume 8, No.6, November - December 2019

International Journal of Advanced Trends in Computer Science and Engineering

Available Online at http://www.warse.org/IJATCSE/static/pdf/file/ijatcse88862019.pdf

https://doi.org/10.30534/ijatcse/2019/88862019

\title{
Performance of Transmission Delay and Energy Consumption of Adaptive Listening Mechanism for Wireless Sensor Networks
}

\author{
Yung-Fa Huang ${ }^{1}$, Jong-Shin Chen ${ }^{1}$, Li-Ming Wang ${ }^{1}$ \\ ${ }^{1}$ Department of Information and Communication Engineering, Chaoyang University of Technology, Taichung, \\ Taiwan,yfahuang@cyut.edu.tw
}

\begin{abstract}
In this paper, we investigated the transmission latency and energy consumption performance of wireless sensor networks (WSNs) without adaptive listening (WOAL) and with adaptive listening (WAL) mechanism. Simulation results show that the transmission delay decreases with the increase of the duty cycle, and the energy consumption increases with the increase of the duty cycle. In addition, comparing with the WOAL, the proposed WAL mechanism can have one more transmission hop and further reduce transmission delay, but does not increase energy consumption.
\end{abstract}

Key words : Wireless sensor network, duty cycle, MAC layer, adaptive listening, transmission hops, energy efficiency.

\section{INTRODUCTION}

With the advancement of micro-electromechanical technology and the development of wireless transmission technology, wireless sensor networks (WSNs) must have low cost, low power consumption, small size, easy layout, and dynamically self-organized [1]. As the technology of WSNs becomes more and more mature, WSNs can be widely used in many different applications, such as health care, military defense, environmental detection, ecological observation, target tracking, and industrial applications, etc. [2, 3, 9]. The WSNs are widely used. However, because the energy of the sensors is supplied by batteries, its radio transmission distance will be limited. In order to solve the energy consumption and distance problems during transmission, if the sensors are too far away from the base station, the WSNs need to organize a large number of distributed nodes to form a multi-hop wireless network.

The sensor uses multiple-hop to establish network routing. The data is transmitted back to the base station through a path composed of multiple sensors [4]. In addition, in a WSN since the wireless sensor is powered by a battery, its energy management is relatively important. There are many methods to reduce energy consumption in WSNs $[5,10]$. Such as path selection when transmitting data, scheduling problems, sleep mechanism when there is no sensitive data, correctness of transmitting data, and so on [11]. This paper mainly discusses its energy efficiency for the Medium Access Control (MAC) layer. In this paper, the performance of the proposed scheduling with adaptive listening (WAL) mechanism is compared with the scheduling without adaptive listening (WOAL) mechanism.

\section{SENSOR-MAC (S-MAC)}

The traditional MAC protocol design goal is to improve the network throughput and reduce the transmission delay while ensuring the fairness of media access. In WSNs, unnecessary energy consumption during data transmission at the MAC layer [5] is mainly in the following areas:

A. Idle listening: Because the node does not know when the data of the neighbor node will be transmitted, it must always keep itself in the mode of receiving data. So it forms an idle listening, which causes unnecessary energy consumption.

B. Collision: If two nodes send data at the same time and interfere with each other, their transmission will fail and the sent packets will be discarded. The energy used to send this packet is relatively wasted at this time.

C. Control Packet: In order to ensure reliable transmission, the protocol will use some control methods, such as RTS/CTS. Although there is no data in it, it must consume a certain amount of energy to send them.

D. Overhearing: Since the wireless channel is a shared medium, the node can also receive data packets that are not to be transmitted to itself, and then discard them, which will also cause energy consumption.

As for how to reduce energy loss, many MAC protocols have proposed corresponding solutions. The most basic method is to keep the node in a sleep state with low energy consumption as much as possible when the node does not need to send data. The sensor MAC (S-MAC) protocol proposes an energy-saving method suitable for multi-hop WSN competition MAC protocol [6]. The S-MAC protocol allows a node to enter the listen and sleep mode periodically. As shown in Fig. 1, a node only receives data in the listening mode, and 
enters sleep in the sleep mode. The S-MAC operation process is shown in Figure 1. When there is data to be transmitted, if the sensor is in the listening state, it can be transmitted directly. When the sensor is in the sleep state, it must be stored in the buffer first. It is transmitted when the sensor status changes to listening.

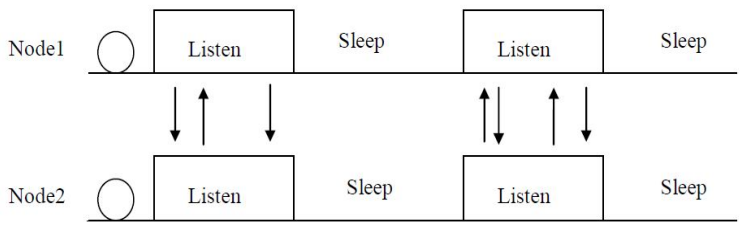

Figure 1: The periodic listening and sleep

The operation of S-MAC is that the transmitting end sends an RTS packet to neighboring nodes before transmitting the data [7]. This RTS frame will contain the time required for the transmitting end to send data. When the neighboring node receives the RTS packet, only The receiver specified in the RTS packet will send a CTS to notify the sender that it can transmit the information. This CTS frame also contains the time required for the transmitter to transmit the resource. The time required for the transmission of the resource is obtained by RTS. Later, it will enter the resource transmission. After the resource is received, the receiving end will send an ACK to tell the transmitting end whether the reception is completed.

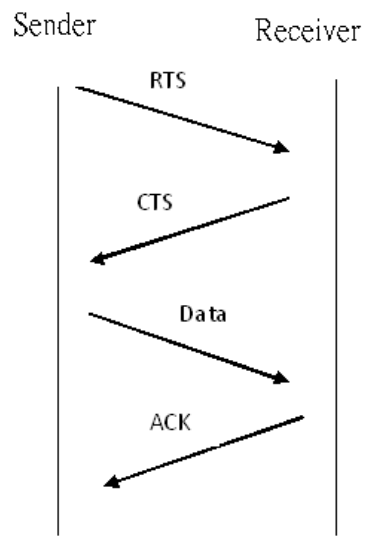

Figure 2: RTS/CTS handshaking protocols

As shown in Fig. 2, the S-MAC can avoid data packet collision and overhearing through the RTS/CTS handshake protocol, and can solve the hidden terminal problem. Although S-MAC periodically enters sleep mode and saves energy, the time spent in listening mode is still a big problem. If the duty cycle is adjusted to be longer, the listening time will be longer, and energy consumption will be for more than idle listening. If the duty cycle is shortened and the listening time is shortened, the delay in data transmission will increase. In addition, adjusting the sensor schedule will also affect the energy consumption and transmission delay. For example, the DMAC in [8] is to adjust the sensor schedule so that each sensor can go to the next sensor immediately after receiving data. Thus it can reduce the delay in data transmission.

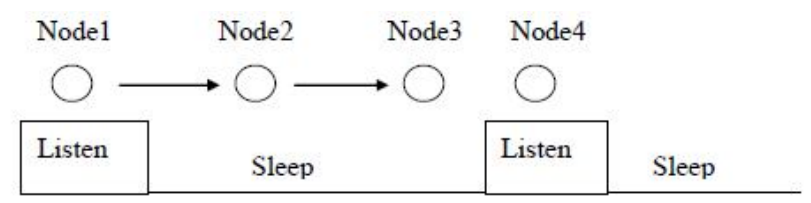

Figure 3: The scheduling with adaptive sleeping (WAL) mechanism

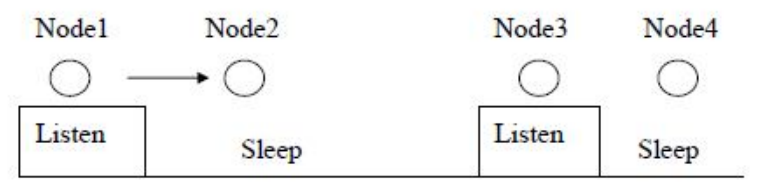

Figure 4: The scheduling without adaptive sleeping (WOAL) mechanism

In order to reduce the delay, an adaptive listening (AL) mechanism is proposed in S-MAC. The basic method of adaptive listening is to make the neighbor nodes hear the RTS or CTS transmitted by the nearby nodes. So the neighbor nodes can wake up for a short time at the end of the transmission. In this way, if the node is the next relay node, the neighbor node can immediately send data to the node, without waiting for the sleep time to wake up to receive the data. As shown in Fig. 3, in the case of WAL, Node3 wakes up at the end of the Node 2 transmission. So it can immediately receive the data of Node2, so the data transmission can end at the first cycle. It does not have to be like Node3 as shown in Fig. 4 for WOAL. It must wait until the second cycle to wake up to receive the data sent by Node2. So the WAL can effectively reduce transmission delay and increase energy efficiency.

\section{SCHEDULING OF MAC}

In WSNs, the mechanism of periodic listening and sleeping in the S-MAC will cause delays. Increasing the duty cycle can reduce the delay but also increase the idle listening time and cause energy consumption. In this Section, we will base on the performance of the performance of S-MAC to adjust the duty cycle to improve the transmission delay and energy consumption.

\subsection{Single Scheduling MAC}

The schedule of each sensor in S-MAC may be different, but we simplified the S-MAC to a fixed schedule. We set one cycle to 1 second. The schedule of ten sensors was set to 0 Second to $T_{\text {on }}$ by the listening state and from $T_{\text {on }}$ to 1.0 second by the sleep state as shown in Figure 5. We compare the transmission delay performance of WOAL and WAL by using computer simulation. In the simulation, we set the first node (Node1) as the source node and the last node (Node10) as the destination node. Figure 5(a) shows that the simulation 
environments of the ten nodes in a line and the distance between each node by 100 meters. We used OMNet network simulator for simulation. The simulation parameters are shown in Table 1 . The duty cycles is set by $3 \%$ to $43 \%$. The whole cycle is 1 second. The packet size is 500 bytes

\subsection{Transmission Delay}

The MAC protocols is simulated according to the CSMA/CA (Carrier sensing multiple access/collision avoid). Before each data transmission, it needs to pass a DIFS contention window $(\mathrm{CW})$, because the next node must receive the RTS to be able to know that there is data to be transmitted. After calculating the time of (DIFS + competition windows+RTS), it is about 0.026 seconds. So the duty cycle must be greater than 0.026 seconds to start the transmission.

Table 1: Simulation Parameters

\begin{tabular}{|c|c|}
\hline Period & $1 \mathrm{sec}$ \\
\hline Duty cycle $(D)$ & $3 \%=0.3 \mathrm{sec} \sim 43 \%=0.43 \mathrm{sec}$ \\
\hline Size of Packet Data & 500 byte $=4000$ bits \\
\hline Bandwidth & 200 Kbps \\
\hline Deployment of nodes & A line \\
\hline Number of Nodes & 10 \\
\hline Adaptive Listening & WOAL, WAL \\
\hline Size of RTS & 160 bits \\
\hline Size of CTS & 112 bits \\
\hline Size of ACK & 112 bits \\
\hline Rounds of Simulation & 20 \\
\hline
\end{tabular}

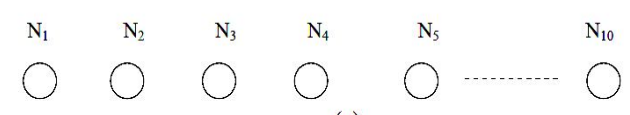

(a)

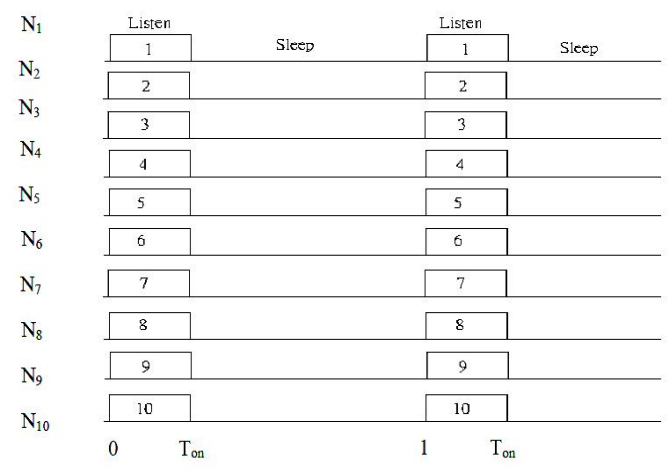

(b)

Figure 5: The WSN deployments of nodes (a)arrangement of $\mathrm{N}_{1}$ to $\mathrm{N}_{10}$ (b)Scheduling diagram of $\mathrm{N}_{1}$ to $\mathrm{N}_{10}$

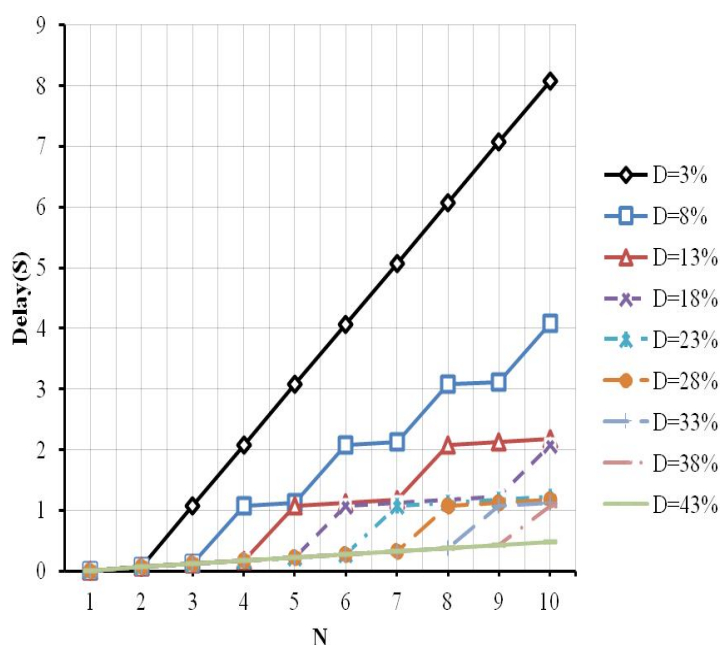

Figure 6: Fix scheduling of WOAL

Therefore, it can see from Fig. 6 that with WOAL and $D=3 \%$, each transmission can only be done once (a Hop). To transmit to the next node, it needs to wait until the next cycle to transmit. The delay between them is about 1 second, so transmission to node 10 takes nine cycles. When D increases to $8 \%$, two hops can be transmitted in one cycle, so it can be transmitted to node 3 in the first cycle. It takes five cycles to transmit to node 10 . We compare $D=3 \%$ and $D=8 \%$ for the tenth node's delay, $D=3 \%$ and $\mathrm{D}=8 \%$ are different by four cycles. So the delay time is about 4 seconds.

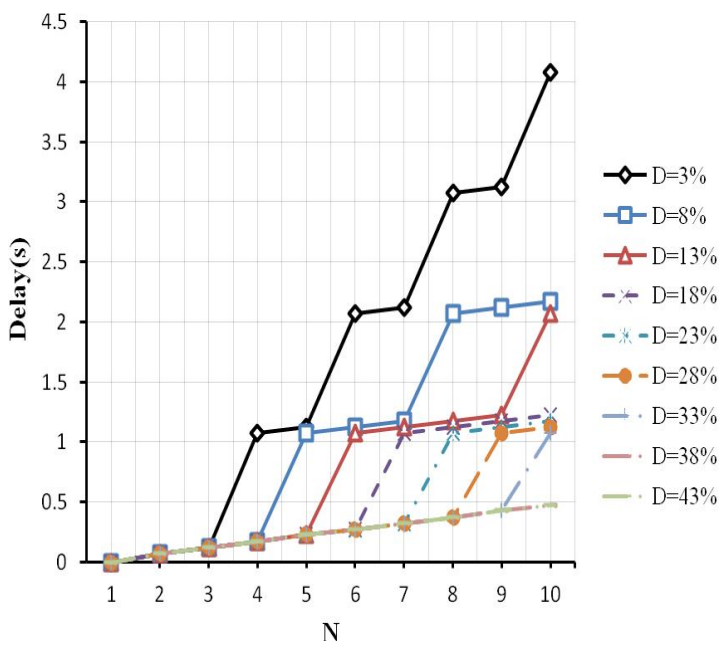

Figure 7: Fix scheduling of WAL

From Fig. 7, it can be observed that with WAL and $D=3 \%$, each transmission can be done twice (two hops). It takes five cycles to transmit to the tenth node. When $D$ increases to $8 \%$, each transmission takes 3 hops. So it only takes three cycles to transmit to the tenth node. Then the delay time between $D=3 \%$ and $D=8 \%$ is about 2 seconds. That is, the proposed WAL has less delay than the WOAL. This is because WAL can transmit one more hop than WOAL in one cycle. 


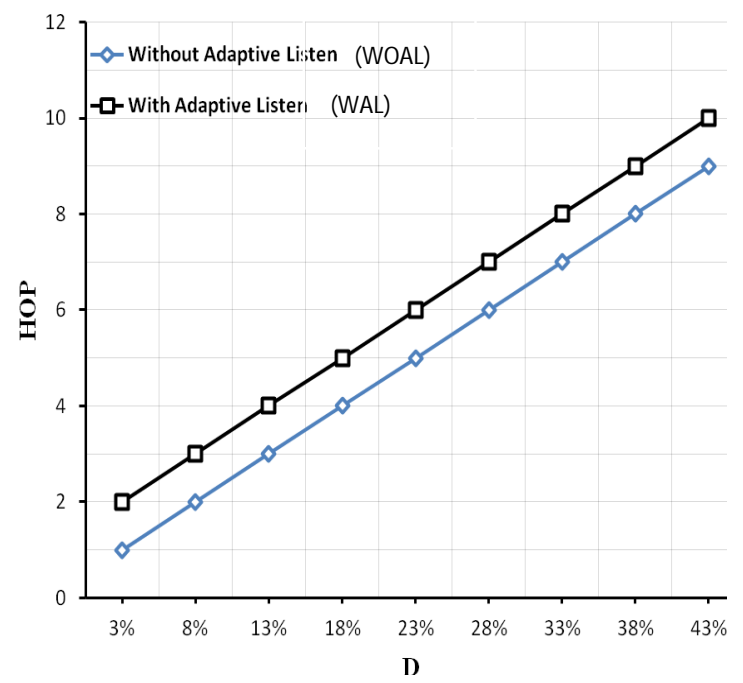

Figure 8: The relationship between WOAL, WAL, with the number of hops for different duty cycles

The relationship between WOAL, WAL, with the number of hops for different duty cycles is shown in Fig. 8. It can be seen that when the duty cycle increases to a certain percentage, the number of hops that a node can transmit in a cycle also gradually increases. In the fixed schedule, we set the schedules of the ten nodes to be the same. When $D=3 \%$ and WOAL, data transmitted with only one Hop in one cycle. So the delay at $D=3 \%$ is relative to other duty cycles. The period is much larger, but after using WAL, the transmission delay at $D=3 \%$ is greatly reduced. From Fig. 8 , it is observed that when $D=3 \%$ is increased to $43 \%$, the WAL transmits one more hop compared to WOAL.

\section{ANALYSIS OF ENERGY CONSUMPTION}

The analysis of energy consumption, there are four parts to discuss. The first part is the energy consumption during data transmission. The second part discusses the energy consumption of the node during a period of transmission. The third part discusses the energy consumption of the idle listening energy consumption of the node without passing through nodes during transmission. The fourth part is the idle listening energy consumption to be transmitted in the last cycle.

\subsection{Energy Consumption of WOAL}

In this Section the energy consumption of WOAL scheme is analyzed. The first part is the energy consumption caused by data transmission. The energy consumption of this part is expressed as

$$
E_{1}=E_{T D} \times(N-1)
$$

where $E_{T D}$ is the energy consumption required for data transmission, including the sum of transmission consumption and reception consumption of RTS, CTS, Data, and ACK. $N$ is the total number of nodes, and $(N-1)$ represents the total number of hops that need to be transmitted.
The second part is the energy consumption of idle listening that will pass through the node in a periodic transmission. It will be expressed as

$$
E_{2}=\left\{\left[\left(T_{D}-T_{t}\right) \times(H-1) \times C_{T}\right]+\left(1+C_{T}\right) \times T_{D}\right\} \times P_{I}
$$

where $T_{D}$ represents the size of the duty cycle, $H$ represents the number of Hops. $C_{T}=\lfloor(N-1) / H\rfloor$ and $P_{I}=E_{\text {elec }} \times l$ that can be transmitted in a cycle, and represents the energy consumed during idle listening. In the case of 500 bytes, the energy consumption of idle listening is $0.2(\mathrm{~mJ})$. The $T_{t}$ can be calculated as 0.0219 seconds.

The third part $E_{3}$ is that the energy consumption of idle listening that does not pass through the node in a period transmission. It can be expressed as

$$
E_{3}=\left\{[N-(H+1)] \times\left\lfloor\frac{N-1}{H}\right\rfloor \times T_{D}\right\} \times P_{I}
$$

where $\mathrm{H}$ represents the number of hops that can be transmitted in a cycle.

The fourth part $E_{4}$ is due to the change in the total number of nodes and the duty cycle, which is caused by some additional idle listening energy consumption expressed by

$$
\begin{aligned}
E_{4}= & {\left[\left(N-\operatorname{Mod}\left(\frac{N-1}{H}\right)\right)-1\right] \times\left(T_{t}+T_{c}\right) \times \operatorname{Mod}\left(\frac{N-1}{H}\right) \times P_{I}(4) } \\
& +\left(T_{D}-T_{t}\right) \times\left[\operatorname{Mod}\left(\frac{N-1}{H}\right)+1\right] \times P_{I}
\end{aligned}
$$

Therefore, we can get the total energy consumption as $E_{\mathrm{c}}=E_{1}+E_{2}+E_{3}+E_{4}$. In the WOAL, the number of hops required

\begin{tabular}{|c|c|c|c|c|c|c|c|c|c|c|c|}
\hline 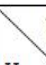 & 10 & 11 & 12 & 13 & 14 & 15 & 16 & 17 & 18 & 19 & 20 \\
\hline 1 & 0 & 0 & 0 & 0 & 0 & 0 & 0 & 0 & 0 & 0 & 0 \\
\hline 2 & 1 & 0 & 1 & 0 & 1 & 0 & 1 & 0 & 1 & 0 & 1 \\
\hline 3 & 0 & 1 & 2 & 0 & 1 & 2 & 0 & 1 & 2 & 0 & 1 \\
\hline 4 & 1 & 2 & 3 & 0 & 1 & 2 & 3 & 0 & 1 & 2 & 3 \\
\hline 5 & 4 & 0 & 1 & 2 & 3 & 4 & 0 & 1 & 2 & 3 & 4 \\
\hline 6 & 3 & 4 & 5 & 0 & 1 & 2 & 3 & 4 & 5 & 0 & 1 \\
\hline 7 & 2 & 3 & 4 & 5 & 6 & 0 & 1 & 2 & 3 & 4 & 5 \\
\hline 8 & 1 & 2 & 3 & 4 & 5 & 6 & 7 & 0 & 1 & 2 & 3 \\
\hline 9 & 0 & 1 & 2 & 3 & 4 & 5 & 6 & 7 & 8 & 0 & 1 \\
\hline 10 & 0 & 0 & 1 & 2 & 3 & 4 & 5 & 6 & 7 & 8 & 9 \\
\hline
\end{tabular}
for the last period of different nodes can be shown as Table 2 for the tenth node to the twentieth node.

Table 2: The number of hops required for the last period of different nodes in WOAL

\subsection{Energy Consumption of WAL}

In S-MAC, the data transmission of the WAL scheme can be passing through one more node than WOAL in a cycle. That is because the WAL has one extra hop for data transmission per cycle. So the energy consumption of the second part is obtained by 


$$
E_{a 2}=\left\{\left[\left(T_{D}-T_{t}\right) \times(H-2) \times C_{T}\right]+\left(1+C_{T}\right) \times T_{D}\right\} \times P_{I}
$$

where $T_{D}$ represents the size of the duty cycle, $H$ represents the number of Hops. $C_{T}=\lfloor(N-1) / H\rfloor$ and $P_{I}=E_{\text {elec }} \times l$ that can be transmitted in a cycle, and represents the energy consumed during idle listening. In the case of 500 bytes, the energy consumption of idle listening is $0.2(\mathrm{~mJ})$. The $T_{t}$ in (1) can be calculated as 0.0219 seconds.

The third part energy consumption $E_{a 3}$ is expressed by

$$
E_{a 3}=\left\{[N-(H+2)] \times\left\lfloor\frac{N-1}{H}\right\rfloor \times T_{D}\right\} \times P_{I}
$$

The fourth part $E_{a 4}$ is due to the change in the total number of nodes and the duty cycle, which is caused by some additional idle listening energy consumption expressed by

$$
\begin{aligned}
E_{a 4}= & {\left[N-\operatorname{Mod}\left(\frac{N-1}{H}\right)-2\right] \times\left(T_{t}+T_{c}\right) \times \operatorname{Mod}\left(\frac{N-1}{H}\right) \times P_{I} } \\
& +\left(T_{D}-T_{t}\right) \times\left[\operatorname{Mod}\left(\frac{N-1}{H}\right)+1\right] \times P_{I}
\end{aligned}
$$

Therefore, we can get the total energy consumption as $E_{a c}=E_{a 1}+E_{a 2}+E_{a 3}+E_{a 4}$. Moreover, In the WAL the number of hops required for the last period of different nodes can be shown as Table 3 for the tenth node to the twentieth node.

Table 3: The number of hops required for the last period of different nodes in WAL

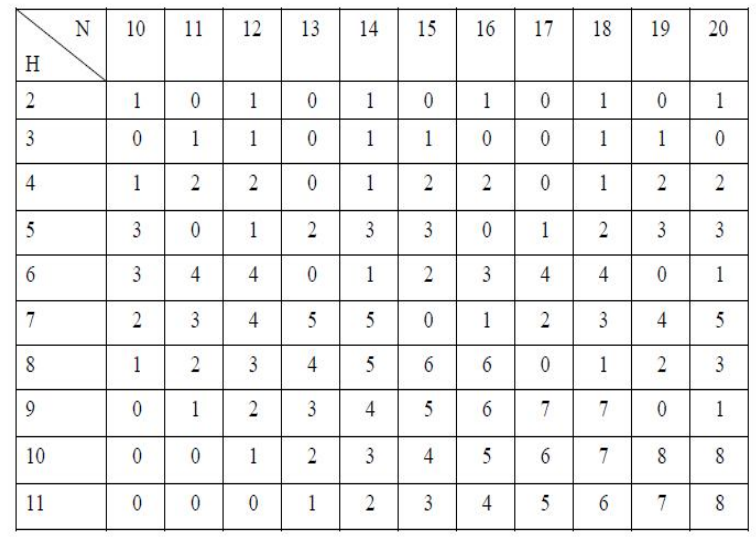

\section{SIMULATION RESULTS}

We compare the energy consumption of WOAL and WAL according to the analysis. When the duty cycle increases, the energy consumption will increase relatively. From Fig. 9, it is seen that it consumes less energy when they reach a certain duty cycle. With $N=10$, there are 9 hops from the source node to the destination node. With $\mathrm{D}=13 \%, 3$ hops can be transmitted in one cycle and the transmission can be completed in the third cycle. On the contrary, it is seen that $D=13 \%$, the energy consumption is less than the energy consumption of $D=8 \%$. With $N=11$ and $D 23 \%$ the energy consumption is less than $D=18 \%$. Because with $D=23 \%$ it is transmitted to the eleventh node and just the second cycle transmission as shown in Table 2. Moreover, with $N=11$ and $D=23 \%$, there is no need to consume any more energy for idle listening in the last cycle. However, with $D=18 \%$, it needs one more cycle. Therefore, it increases energy consumption. Then with $D=23 \%$, it actually consumes less energy than $D=18 \%$.

We adjusted the number of nodes and the duty cycle to simulate the situation of observing nodes in the WAL. From Fig. 10, it is observed four lines which consumed less energy when they reach a certain duty cycle. When $N=10$, there are 8 hops from the source node to the destination node. When $D=8 \%, 3$ hops can be transmitted in one cycle and the transmission can be completed in the third cycle. On the contrary, it is seen that with $D=8 \%$ the energy consumption is less than the energy consumption of $D=3 \%$. When $N=11$, energy consumption with $D=18 \%$ is less than the energy consumption with $D=13 \%$. This is because that when $D=18 \%$ the transmission to the eleventh node is just the second cycle transmission. It can be seen that from Table 3, with $N=11$, $\mathrm{D}=18 \%$, there is no need to consume any more energy for idle listening in the last cycle. But with $D=13 \%$, it need one more cycle. Therefore, with $D=18 \%$ it actually consumes less energy than $D=13 \%$.

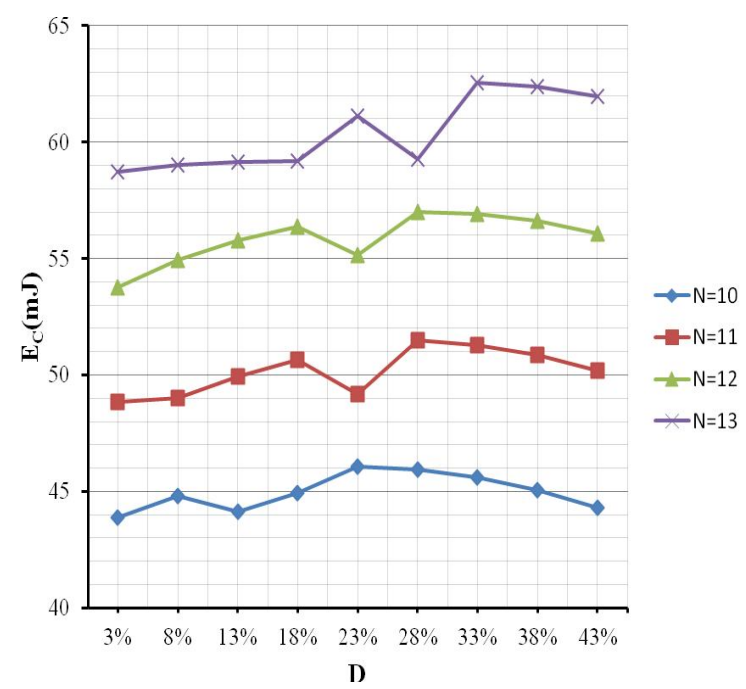

Figure 9: Relationship between responsibility cycle and energy consumption for WOAL algorithms 


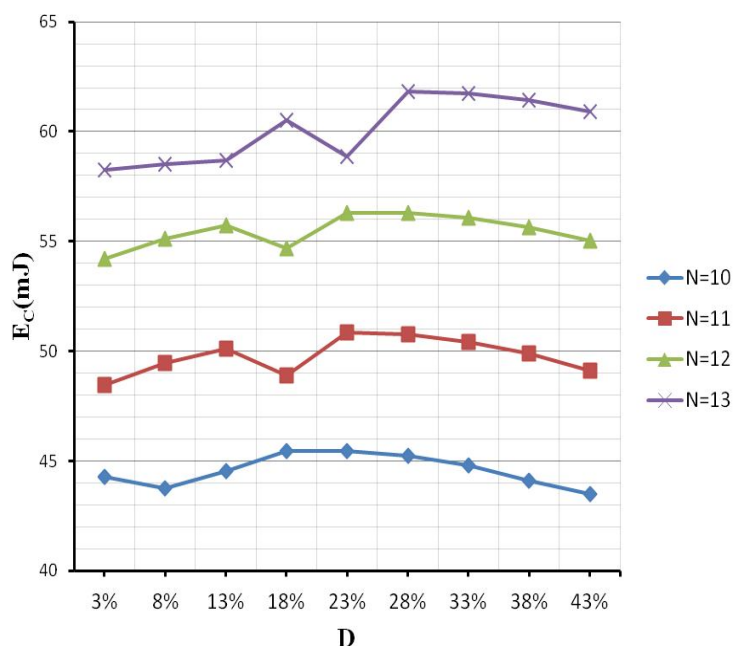

Figure 10: Relationship between responsibility cycle and energy consumption for WAL algorithms

\section{CONCLUSION}

In this paper, we adjusted the schedule of each node to a fixed listening state by 0.03 seconds to 0.43 seconds. Simulation results showed that the proposed WAL scheme can outperform the WOAL scheme for the transmission delay and energy consumption.

\section{ACKNOWLEDGEMENT}

The results of this research are sponsored by the Ministry of Science and Technology (MOST), Taiwan, with the grant number is MOST 108-2221- E-324- 010-.

\section{REFERENCES}

1. I. F. Akyildiz, W. Su, Y. Sankarasubramaniam, and E. Cayirci. A survey on sensor networks, IEEE Communication Magazine, vol. 42, no. 8, pp. 102-114, Aug. 2002.

https://doi.org/10.1109/MCOM.2002.1024422

2. Q. Dong, and W. Dargie. A survey on mobility and mobility-aware MAC protocols in wireless sensor networks, IEEE Communications Survey \& Tutorials, vol. 15, no. 1, pp. 88-100, Jan. 2013. https://doi.org/10.1109/SURV.2012.013012.00051

3. C. C. Enz, A. El-Hoiydi, J.D. Decotignie, and V. Peiris. WiseNET: An ultralow-power wireless sensor network solution, IEEE Computer., vol. 37, pp. 62-70, Aug. 2004.

https://doi.org/10.1109/MC.2004.109

4. J. N. Al-Karaki and A. E. Kamal. Routing techniques in wireless sensor networks: A survey, IEEE Wireless Communications, vol. 20, no. 6, pp. 6-28, Dec. 2004. https://doi.org/10.1109/MWC.2004.1368893

5. W. Ye, J. Heidemann, and D. Estrin, Medium access control with coordinated adaptive sleeping for wireless sensor networks. IEEE/ACM Trans. on Networking, vol. 12, pp. 493-506, June 2004. https://doi.org/10.1109/TNET.2004.828953

6. D. Kim, J. Jung, Y. Koo, and Y. Yi. Revisiting sensor MAC for periodic monitoring: Why should transmitters be early birds? in Proc. 2017 14th Annual IEEE International Conference on Sensing, Communication, and Networking (SECON), San Diego, CA, USA,12-14 June 2017, pp. 1-4. https://doi.org/10.1109/SAHCN.2017.7964936

7. J. Zhu and S. Papavassiliou, On the energy-efficient organization and the lifetime of multi-hop sensor networks. IEEE Communication Letters, vol. 7, pp. 537-539, Nov. 2003.

https://doi.org/10.1109/LCOMM.2003.820097

8. S. Lindsey, C. Raghavendra and K. M. Sivalingam. Data gathering algorithms in sensor networks using energy metrics, IEEE Trans. on Parallel and Distributed Systems, vol. 13, no. 9, pp. 924-935, Sept. 2002. https://doi.org/10.1109/TPDS.2002.1036066

9. S.V.R.K. Rao, M. Saritha Devi, A.R. Kishore and Praveen Kumar. Wireless sensor network based industrial automation using internet of things (IoT), International Journal of Advanced Trends in Computer Science and Engineering, vol. 7, no. 6, pp. 82-86, November- December 2018.

https://doi.org/10.30534/ijatcse/2018/01762018

10. N. S. Alotaibi. An efficient technique for reducing transmission energy of sensor node to enhance the lifetime of the WSN's, International Journal of Advanced Trends in Computer Science and Engineering, vol. 8, no.2, March - April 2019.

https://doi.org/10.30534/ijatcse/2019/02822019

11. A. N. Rao, B. R. Naik, and L. N. Devi. Location aware relay node placement for energy constrained wireless sensor networks, International Journal of Advanced Trends in Computer Science and Engineering, vol. 8, no.3, pp. 519-526, May - June 2019. https://doi.org/10.30534/ijatcse/2019/28832019 